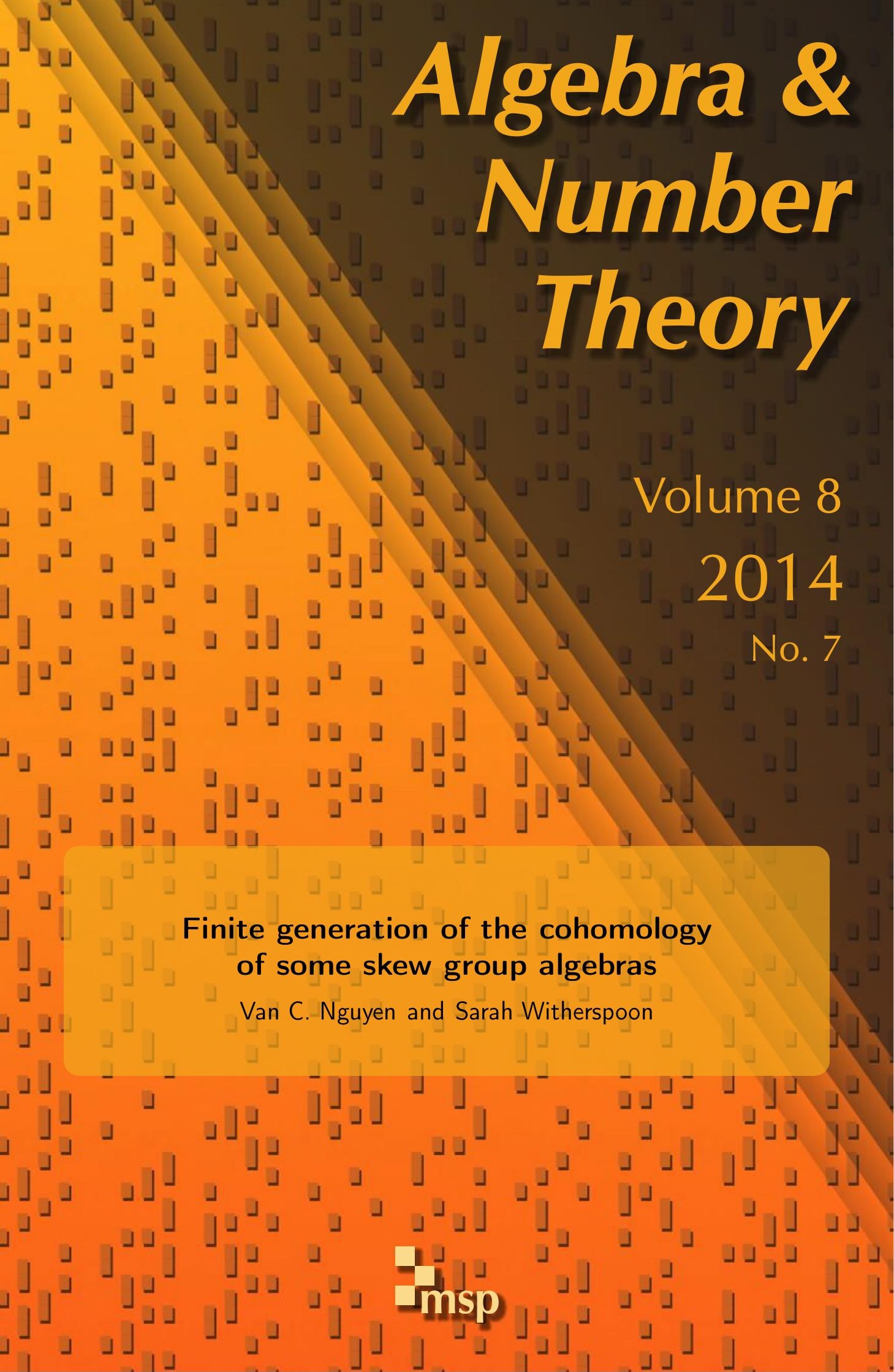




\title{
Finite generation of the cohomology of some skew group algebras
}

\author{
Van C. Nguyen and Sarah Witherspoon
}

\begin{abstract}
We prove that some skew group algebras have Noetherian cohomology rings, a property inherited from their component parts. The proof is an adaptation of Evens' proof of finite generation of group cohomology. We apply the result to a series of examples of finite-dimensional Hopf algebras in positive characteristic.
\end{abstract}

\section{Introduction}

The cohomology ring of a Hopf algebra encodes potentially useful information about its structure and representations. It is always graded commutative (see, for example, [Suarez-Alvarez 2004]). For many classes of finite-dimensional Hopf algebras, it is also known to be finitely generated: for example, cocommutative Hopf algebras [Friedlander and Suslin 1997], small quantum groups [Ginzburg and Kumar 1993], and small quantum function algebras [Gordon 2000]. Etingof and Ostrik [2004] conjectured that it is always finitely generated, as a special case of a conjecture about finite tensor categories. Snashall and Solberg [2004] made an analogous conjecture for Hochschild cohomology of finite-dimensional algebras that was seen to be false when $\mathrm{Xu}$ [2008] constructed a counterexample. In contrast, there is neither a counterexample nor a proof of the Hopf algebra conjecture. Each finite generation result so far has used, in crucial ways, known structure of a particular class of Hopf algebras. Further progress will require new ideas.

In this article, we present one technique for handling some types of algebras inductively. Many (Hopf) algebras of interest are skew group algebras (that is, smash products with group algebras). Under some conditions on a skew group algebra, we show that its cohomology is Noetherian if the same is true of the underlying algebra on which the group acts.

This material is based upon work done while Nguyen was a Texas A\&M graduate student. It was supported by the National Science Foundation under grant No. 0932078000 while both Nguyen and Witherspoon were in residence at the Mathematical Sciences Research Institute (MSRI) in Berkeley, California, during the semester of Spring 2013. Both authors were also supported by NSF grant DMS-1101399.

MSC2010: primary 16E40; secondary 16T05.

Keywords: cohomology, Hopf algebras, skew group algebras. 
Specifically, if $A$ is a finite-dimensional augmented algebra over a field $k$, with an action of a finite group $G$ by automorphisms, there is a spectral sequence relating the cohomology of the smash product $A \# k G$ (definition in Section 2) as an augmented algebra to that of each of $A$ and $G$. (It is essentially the Lyndon-Hochschild-Serre spectral sequence.) This allows us to use the framework of Evens' classic proof [1961] of finite generation of group cohomology to prove that the cohomology rings of some smash products are Noetherian (Theorem 3.1). In order to do this, we need a particularly nice set of permanent cycles in the cohomology of $A$. In the finite group case, these cycles exist due to an application of Evens' norm map. In our setting, there may be no such norm map, and we instead hypothesize existence of these permanent cycles.

We focus on a class of examples (in Section 5) found by Cibils, Lauve, and the second author [Cibils et al. 2009] that satisfy our hypotheses. We prove finite generation of the cohomology of these noncommutative, noncocommutative Hopf algebras in positive characteristic. While our main theorem is tailored to suit these examples, we state and prove it in the abstract setting, in order to add one more tool to the collection of techniques available for proving finite generation. Our restrictive hypotheses serve to highlight the difficulty in adapting methods designed for the finite group setting, where serendipity reigns.

\section{Definitions and notation}

Throughout this article, let $k$ be a field. All algebras will be associative algebras over $k$, and all modules will be left modules, finite-dimensional over $k$. Let $\otimes=\otimes_{k}$.

Let $G$ be a finite group acting on a finite-dimensional augmented $k$-algebra $A$ by automorphisms. Let $A \# k G$ be the resulting smash product (or skew group algebra), that is, $A \otimes k G$ as a vector space, with multiplication $(a \otimes g)(b \otimes h)=a\left({ }^{g} b\right) \otimes g h$, for all $a, b \in A$ and $g, h \in G$. (For simplicity, we will drop tensor symbols in this notation from now on.) We assume the action of $G$ preserves the augmentation of $A$, so that $A \# k G$ is also augmented with augmentation map $\varepsilon_{A \# k G}: A \# k G \rightarrow k$ defined by $\varepsilon_{A \# k G}(a g)=\varepsilon_{A}(a)$, for all $a \in A, g \in G$.

We use the symbol $k$ also to denote the one-dimensional $A$-module (respectively, $A \# k G$-module) on which $A$ (respectively, $A \# k G$ ) acts via its augmentation. Let

$$
\mathrm{H}^{*}(A, k):=\operatorname{Ext}_{A}^{*}(k, k) \text { and } \mathrm{H}^{*}(A \# k G, k):=\operatorname{Ext}_{A \# k G}^{*}(k, k) .
$$

Both are algebras under Yoneda composition. The embedding of $A$ into $A \# k G$ as a subalgebra induces a restriction map

$$
\operatorname{res}_{A \# k G, A}: \mathrm{H}^{*}(A \# k G, k) \rightarrow \mathrm{H}^{*}(A, k)
$$

on cohomology. There is an action of $G$ on $\mathrm{H}^{*}(A, k)$ that may be defined for example via the diagonal action of $G$ on the components of the bar resolution for $A$. 
There is a similar action of $G$ on $\mathrm{H}^{*}(A \# k G, k)$ that is trivial since it comes from inner automorphisms on $A \# k G$.

\section{Finite generation of cohomology}

In this section, we prove our main theorem that under certain hypotheses, the cohomology ring $\mathrm{H}^{*}(A \# k G, k)$ of $A \# k G$ is Noetherian:

Theorem 3.1. Let $G$ be a finite group acting on a finite-dimensional augmented algebra A, preserving the augmentation map. Assume that $\operatorname{Im}\left(\operatorname{res}_{A \# k G, A}\right)$ contains a polynomial subalgebra over which $\mathrm{H}^{*}(A, k)$ is Noetherian and free as a module, with a free basis whose $k$-linear span is a $k G$-submodule of $\mathrm{H}^{*}(A, k)$. Then, $\mathrm{H}^{*}(A \# k G, k)$ is Noetherian.

Remarks 3.2. (a) The hypothesis that $\operatorname{Im}\left(\operatorname{res}_{A \# k G, A}\right)$ contains a polynomial subalgebra over which $\mathrm{H}^{*}(A, k)$ is Noetherian, together with the left module version of [Goodearl and Warfield 2004, Corollary 1.5], implies that $\mathrm{H}^{*}(A, k)$ is (left) Noetherian.

(b) We did not specify the characteristic of the base field $k$ in the theorem. If the characteristic of $k$ does not divide the order of $G$, then $k G$ is semisimple and its cohomology is trivial except in degree zero. In this case, $\mathrm{H}^{*}(A \# k G, k) \cong \mathrm{H}^{*}(A, k)^{G}$, the invariant ring under the action of $G$. Here, one can use invariant ring theory in the noncommutative setting to show that the conclusion of the theorem holds. (See, for example, [Montgomery 1993, Corollary 4.3.5].) For the proof of Theorem 3.1, we assume the characteristic of $k$ divides the order of $G$.

Proof. We use the Lyndon-Hochschild-Serre spectral sequence (see, for example, [Barnes 1985, Chapter VI] in a very general setting):

$$
E_{2}^{p, q}=E_{2}^{p, q}(k)=\mathrm{H}^{p}\left(G, \mathrm{H}^{q}(A, k)\right) \Longrightarrow \mathrm{H}^{p+q}(A \# k G, k) .
$$

Let $E_{r}(k)$ denote the resulting $r$-th page, and note that for each $q, \mathrm{H}^{q}(A, k)$ is a finite-dimensional $k$-vector space.

Note that $E_{\infty}^{0, *}$ is a submodule of $E_{2}^{0, *}$, since no $d_{r}: E_{r}^{p, q} \rightarrow E_{r}^{p+r, q-r+1}$ ends on the vertical edge. It follows that the restriction map $\mathrm{H}^{*}(A \# k G, k) \rightarrow E_{2}^{0, *}(k)$ is part of the following commuting diagram:

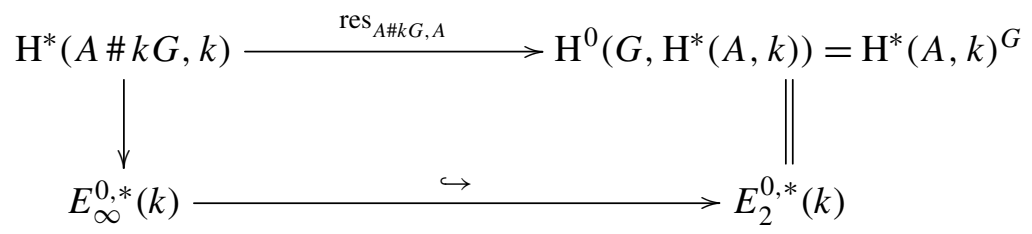

We can identify $E_{\infty}^{0, *}$ with the image of the restriction map in $E_{2}^{0, *}$. 
Let $T=k\left[\chi_{1}, \ldots, \chi_{m}\right]$ denote the polynomial subalgebra of $\operatorname{Im}\left(\operatorname{res}_{A \# k G, A}\right)$ hypothesized in the statement of the theorem. The action of $G$ on $\mathrm{H}^{*}(A, k)$ restricts to the trivial action on $T$ since it is a subalgebra of $\operatorname{Im}\left(\right.$ res $\left._{A \# k G, A}\right)$. Therefore, by the universal coefficients theorem, $\mathrm{H}^{*}(G, T) \cong \mathrm{H}^{*}(G, k) \otimes T$, an isomorphism of graded algebras.

Let $S:=\mathrm{H}^{*}(G, k)=E_{2}^{*, 0}(k)$. Let $R$ be the subring of $E_{2}(k)$ generated by $S$ and $T$. By the above observations, $R \cong S\left[\chi_{1}, \ldots, \chi_{m}\right]$, a polynomial ring over $S$ in $m$ indeterminates (that we also denote by $\chi_{1}, \ldots, \chi_{m}$ for convenience). Since $d_{2}$ vanishes on the horizontal edge, $R \subseteq \operatorname{Ker}\left(d_{2}\right)$. So $R$ projects onto a subring of $E_{3}(k)=\mathrm{H}\left(E_{2}(k), d_{2}\right)$. Similarly, $R$ projects onto a subring of $E_{r}(k)$ for every $r>0$ including $\infty$. Therefore, we may consider $E_{r}(k)$ to be a module over $R$, for every $r>0$ including $\infty$.

Claim 1. $E_{2}(k)$ is a Noetherian module over $R$.

Proof of Claim 1. By hypothesis, there are (homogeneous) elements $\rho_{1}, \ldots, \rho_{t} \in$ $\mathrm{H}^{*}(A, k)$ that form a free basis of $\mathrm{H}^{*}(A, k)$ as a $T$-module, and for which

$$
V:=\operatorname{Span}_{k}\left\{\rho_{1}, \ldots, \rho_{t}\right\}
$$

is a $k G$-submodule of $\mathrm{H}^{*}(A, k)$. Let

$$
L:=\mathrm{H}^{*}(G, V) .
$$

Note that $L$ contains a copy of $S=\mathrm{H}^{*}(G, k)$ as $V$ must include an element in degree 0 , that is, in $\mathrm{H}^{0}(A, k) \cong k$, which has trivial $G$-action. By hypothesis, $\mathrm{H}^{*}(A, k)=k\left[\chi_{1}, \ldots, \chi_{m}\right] \cdot V$, and so

$$
E_{2}(k)=\mathrm{H}^{*}\left(G, k\left[\chi_{1}, \ldots, \chi_{m}\right] \cdot V\right) .
$$

Further, $k\left[\chi_{1}, \ldots, \chi_{m}\right]$ has trivial $G$-action and the module $\mathrm{H}^{*}(A, k)$ for this polynomial ring is free with free basis $\rho_{1}, \ldots, \rho_{t}$. It follows that, as a $k G$-module,

$$
k\left[\chi_{1}, \ldots, \chi_{m}\right] \cdot V \cong \bigoplus_{i_{1}, \ldots, i_{m} \geq 0} \chi_{1}^{i_{1}} \cdots \chi_{m}^{i_{m}} \cdot V \cong \bigoplus_{i_{1}, \ldots, i_{m} \geq 0} V
$$

a direct sum of copies of the same $k G$-module, $V$. Therefore by the universal coefficients theorem, $E_{2}(k)$ is the image of

$$
\mathrm{H}^{0}\left(G, k\left[\chi_{1}, \ldots, \chi_{m}\right]\right) \otimes \mathrm{H}^{*}(G, V) \cong k\left[\chi_{1}, \ldots, \chi_{m}\right] \otimes L,
$$

under cup product. We thus identify $E_{2}^{* * *}(k)$ with $S\left[\chi_{1}, \ldots, \chi_{m}\right] \otimes_{S} L$.

Since $G$ is a finite group and $V$ is a finite-dimensional vector space over $k$, $L=\mathrm{H}^{*}(G, V)$ is Noetherian over $S=\mathrm{H}^{*}(G, k)$ [Evens 1961]. By the Hilbert basis theorem for graded commutative rings (see, for example, [Goodearl and Warfield 2004, Theorem 2.6]), $S\left[\chi_{1}, \ldots, \chi_{m}\right] \otimes_{S} L$ is Noetherian over $R=S\left[\chi_{1}, \ldots, \chi_{m}\right]$. 
Therefore, $E_{2}^{*, *}(k)$ is Noetherian over $R$. We have proven Claim 1.

Claim 2. The spectral sequence stops; i.e., $E_{r}=E_{\infty}$ for some $r<\infty$.

Proof of Claim 2. Let $Z_{i}$ be the space of $i$-cocycles and $B_{i}$ be the space of $i$ coboundaries in $E_{i}=E_{i}(k)$. Recall that $E_{1}=Z_{1}$ and $E_{2}=Z_{2} / B_{2}$. Consider the "pull back" $B_{r}$ in $E_{2}$ of $d_{r}\left(E_{r}\right)$ as follows.

Each element of $E_{2}$ on which $d_{2}$ vanishes determines an element of $E_{3}$. Suppose $d_{3}$ vanishes on that element, so that it in turn determines an element of $E_{4}$. Continue placing such restrictions until we determine an element of $E_{r}$, and suppose that element is in the image of $d_{r}$. We define:

$$
B_{r}:=\left\{\tau \in E_{2}: \tau \in \operatorname{Ker}\left(d_{i}\right) \text { for } 2 \leq i \leq r-1 \text { and } \tau \in \operatorname{Im}\left(d_{r}\right)\right\} .
$$

Note that $B_{r}$ is an $R$-submodule of $E_{2}$ since $d_{j}$ is a derivation for all $j, 2 \leq j \leq r$, and the image in each $E_{j}$ of $R$ consists of universal cycles. Moreover, $B_{r} \subseteq B_{r+1}$ so we obtain an ascending chain of $R$-submodules of $E_{2}$ :

$$
0=B_{1} \subseteq B_{2} \subseteq \cdots
$$

Since $E_{2}$ is Noetherian over $R$ by Claim 1, this chain must stabilize by the ascending chain condition. Thus there exists some $r_{0}$ finite such that $B_{r_{0}}=B_{r_{0}+1}=B_{r_{0}+2}=\cdots$, and so $d_{r}=0$ for all $r>r_{0}$. This implies $E_{r}=E_{\infty}$ for $r>r_{0}$, proving Claim 2 .

We can put this together to finish the proof of the theorem: Each $Z_{r}, B_{r}$ is a submodule of $E_{2}$ over $R=S\left[\chi_{1}, \ldots, \chi_{m}\right]$. Thus, each $E_{r}$, which is a submodule of a quotient module of $E_{r-1}$, is Noetherian over $R$ by Claim 1 and induction on $r$. By Claim 2, $E_{\infty}$ is Noetherian over $R$, and so by [Goodearl and Warfield 2004, Corollary 1.5] it is a Noetherian ring.

Now, $\mathrm{H}^{*}(A \# k G, k)$ has a filtration whose filtered quotients are

$$
E_{\infty}^{p, q}(k) \cong \frac{F^{p} \mathrm{H}^{p+q}(A \# k G, k)}{F^{p+1} \mathrm{H}^{p+q}(A \# k G, k)} .
$$

Suppose that $\mathrm{H}^{*}(A \# k G, k)$ is not Noetherian and let $T_{1} \subseteq T_{2} \subseteq \cdots \subseteq \mathrm{H}^{*}(A \# k G, k)$ be an infinite ascending chain of ideals of $\mathrm{H}^{*}(A \# k G, k)$. Let

$$
F^{p} T_{i}:=T_{i} \cap F^{p} \mathrm{H}^{*}(A \# k G, k)
$$

and

$$
U_{i}:=\bigoplus_{p \geq 0} F^{p} T_{i} / F^{p+1} T_{i} \subseteq E_{\infty}(k) .
$$

If $x \in T_{i+1} \backslash T_{i}$, then for some $p, x \in F^{p} T_{i+1}$ but $x \notin F^{p} T_{i}$ and $x \notin F^{p+1} T_{i+1}$, so $x+F^{p+1} T_{i+1}$ is not in the image of the inclusion

$$
F^{p} T_{i} / F^{p+1} T_{i} \hookrightarrow F^{p} T_{i+1} / F^{p+1} T_{i+1},
$$


that is, $x \in U_{i+1} \backslash U_{i}$. So $U_{i+1}$ properly contains $U_{i}$, for all $i$. Therefore, we have an infinite ascending chain of ideals of $E_{\infty}(k)$ :

$$
U_{1} \varsubsetneqq U_{2} \varsubsetneqq \cdots
$$

This contradicts the result that $E_{\infty}(k)$ is Noetherian. Therefore, $\mathrm{H}^{*}(A \# k G, k)$ is Noetherian.

Remark 3.3. Theorem 3.1 parallels the main step in Evens' proof of finite generation of group cohomology: Let $H$ be a finite $p$-group (where $k$ has characteristic $p$ ), $A=k Z$ is the group algebra of a central subgroup $Z$ of $H$ of order $p$, and $G=H / Z$. (In case $Z$ is complemented in $H$, we obtain $k H \cong A \# k G$, whereas more generally, $k H$ is a crossed product of $A$ with $G$.) In this case, Evens' norm map is applied to show that $\operatorname{Im}\left(\operatorname{res}_{k H, k Z}\right)$ contains a polynomial subalgebra $k[\zeta]$ (in one indeterminate). One observes that $\mathrm{H}^{*}(k Z, k)$ is a free module over $k[\zeta]$, and that the $k$-linear span of any free basis is a $k G$-submodule. This special case is somewhat simpler than our more general context as it uses a polynomial ring in one indeterminate.

We are particularly interested in those actions of finite groups $G$ on algebras $A$ for which $A \# k G$ is a Hopf algebra. We turn to a class of such examples in the remainder of the paper.

\section{Examples: Nichols algebras in positive characteristic}

In this section, we first recall the Nichols algebras $A$ from [Cibils et al. 2009, Corollary 3.14] and the corresponding Hopf algebras $A \# k G$ from the same paper. We will prove that these Hopf algebras have finitely generated cohomology. This will follow from Theorem 3.1 and explicit calculation using Anick's resolution [1986]. In this section we explain these calculations for $A$, and in the next we complete the proof of finite generation of cohomology of $A \# k G$. The results of this section were anticipated by $\varnothing$. Solberg (personal communication, 2012) as a consequence of computer calculations (for small $p$ ) that gave the graded vector space structure and generators of cohomology.

In the remainder of the paper, $k$ will be a field of characteristic $p>2$. (The case $p=2$ is included in [Cibils et al. 2009], but is different, and we will not consider that case here.) Let $A$ be the augmented $k$-algebra generated by $a, b$, with relations

$$
a^{p}=0, \quad b^{p}=0, \quad b a=a b+\frac{1}{2} a^{2},
$$

and augmentation $\varepsilon: A \rightarrow k$ given by $\varepsilon(a)=\varepsilon(b)=0$. Let $G$ be a cyclic group of order $p$ with generator $g$, acting on $A$ by

$$
g(a)=a, \quad g(b)=b-a .
$$


Then $A \# k G$ is a Hopf algebra with comultiplication given by

$$
\Delta(g)=g \otimes g, \quad \Delta(a)=a \otimes 1+g \otimes a, \quad \Delta(b)=b \otimes 1+g \otimes b .
$$

It is useful to consider $A$ as a quotient of a larger algebra. Let

$$
B:=k\langle a, b\rangle /\left(b a-a b-\frac{1}{2} a^{2}\right),
$$

so that $A \cong B /\left(a^{p}, b^{p}\right)$. We will show that $B$ is a PBW algebra in the sense of [Bueso et al. 2003] or [Shroff 2013, Section 2], although we will not need this fact for our cohomology calculations.

Choose the lexicographic order on $\mathbb{N}^{2}$ for which $(0,1)<(1,0)$, and assign $\operatorname{deg}(a)=(0,1), \operatorname{deg}(b)=(1,0)$. Then $b a-a b-\frac{1}{2} a^{2}$ is a Gröbner basis for the ideal of the free algebra $k\langle a, b\rangle$ that it generates. It follows that $\left\{a^{i} b^{j} \mid i, j \geq 0\right\}$ is a vector space basis of $B$. The relation $a b=b a-\frac{1}{2} a^{2}$ satisfies the required condition in the definition of a PBW algebra since $\operatorname{deg}\left(a^{2}\right)<\operatorname{deg}(a b)$, so $B$ is a PBW algebra. Moreover, $B$ is a Koszul algebra by Theorem 5.3 in [Priddy 1970].

Applying [Cibils et al. 2009, (3.9)], one finds that the elements $a^{p}, b^{p}$ are in the center of $B$. We may thus apply Theorem 4.3 of [Shroff 2013] to the Nichols algebra $A$ to conclude that the cohomology ring $\mathrm{H}^{*}(A, k)$ of $A$ is Noetherian.

We will need some details about this cohomology of $A$ for the next section. For this, we will construct Anick's resolution [1986] for $A$, and show that it is minimal. We use the combinatorial description of the resolution given by Cojocaru and Ufnarovski [1997], however we index differently, and use left modules instead of right. This is a free resolution of the trivial $A$-module $k$, of the form

$$
\cdots \longrightarrow A \otimes k C_{2} \longrightarrow A \otimes k C_{1} \longrightarrow A \otimes k C_{0} \longrightarrow k \rightarrow 0
$$

for (finite) sets $C_{n}$, where $k C_{n}$ denotes the vector space with basis $C_{n}$. Let $C_{0}:=\{1\}$ and $C_{1}:=\{a, b\}$. Then $C_{2}:=\left\{a^{p}, b^{p}, b a\right\}$ is the set of "tips" or "obstructions." To define $C_{n}$ in general, consider the graph

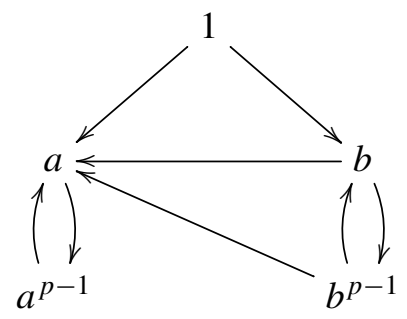

The elements of $C_{n}$ correspond to paths of length $n$ that start at 1 . We label such paths with the product of all elements through which the path passes (including the 
endpoint). In this way we obtain

$$
\begin{aligned}
& C_{3}=\left\{a^{p+1}, b^{p+1}, b^{p} a, b a^{p}\right\}, \\
& C_{4}=\left\{a^{2 p}, b^{2 p}, b^{p+1} a, b^{p} a^{p}, b a^{p+1}\right\},
\end{aligned}
$$

and in general

$$
\begin{aligned}
C_{2 m-1} & =\left\{b^{k p} a^{(m-1-k) p+1}, b^{k p+1} a^{(m-1-k) p} \mid k=0,1, \ldots, m-1\right\}, \\
C_{2 m} & =\left\{b^{m p}, b^{k p} a^{(m-k) p}, b^{k p+1} a^{(m-1-k) p+1} \mid k=0,1, \ldots, m-1\right\} .
\end{aligned}
$$

For qualitative understanding of the differentials, give each of the generators $a, b$ of $A$ the degree 1 . We claim that the differentials preserve degree, where the graded module structure of a tensor product $A \otimes k C_{i}$ is given by $\operatorname{deg}(a \otimes x)=$ $\operatorname{deg}(a)+\operatorname{deg}(x)$ if $a, x$ are homogeneous. This claim results from the recursive definition of the differential $d$ in each homological degree: By construction, $d$ applied to elements of $A \otimes k C_{1}$ is multiplication, and to $A \otimes k C_{2}$ takes each tip to the Gröbner basis element to which it corresponds, suitably expressed as an element of $A \otimes k C_{1}$. The remaining differentials are defined iteratively, via splitting maps in each homological degree that are also defined iteratively. Since the relations are homogeneous and differentials in low homological degrees preserve degrees of elements, the splitting maps and differentials in higher degrees may be chosen to have the same property.

Now note that $C_{2 m-1}$ consists of elements of degree $(m-1) p+1$, and $C_{2 m}$ consists of elements of degrees $m p$ and $(m-1) p+2$. Therefore elements of $C_{n}$ and of $C_{n-1}$ never have the same degree. As a consequence the differential takes elements of $C_{n}$ to elements of $A_{+} \otimes C_{n-1}$, where $A_{+}$denotes all elements of $A$ of positive degree (and these are in the kernel of the augmentation map $\varepsilon$ ). When applying the functor $\operatorname{Hom}_{A}(-, k)$, then, the induced differentials all become 0 . Therefore in this case, Anick's resolution is minimal, and for each $n$, the dimension of $\mathrm{H}^{n}(A, k)$ is $n+1$.

\section{Examples: pointed Hopf algebras in positive characteristic}

We wish to apply Theorem 3.1 to the Hopf algebras $A \# k G$ introduced in the previous section. In order to do this, we next give some of the details from [Shroff 2013, Section 4] as they apply to these examples in particular. Recall the PBW algebra $B$ defined in (4-1). Let $\xi_{a}, \xi_{b}: B \otimes B \rightarrow k$ be the $k$-linear functions given by

$$
\xi_{a}(r \otimes s)=\gamma_{a}, \quad \xi_{b}(r \otimes s)=\gamma_{b},
$$

where $\gamma_{a}$ and $\gamma_{b}$ are the scalar coefficients of $a^{p}$ and $b^{p}$, respectively, in the product $r s$ in $B$. (Shroff writes these functions $\widetilde{\zeta}_{1}, \widetilde{\zeta}_{2}$.) Extending to left $B$-module homomorphisms in $\operatorname{Hom}_{B}\left(B^{\otimes 3}, k\right)$ under the isomorphism $\operatorname{Hom}_{B}\left(B^{\otimes 3}, k\right) \cong \operatorname{Hom}_{k}\left(B^{\otimes 2}, k\right)$, 
the functions $\xi_{a}, \xi_{b}$ are coboundaries on the bar resolution of $B$, as shown in [loc. cit.], and they factor through $A \cong B /\left(a^{p}, b^{p}\right)$. The resulting functions (which we will also denote $\xi_{a}, \xi_{b}$ by abuse of notation) are no longer coboundaries. They represent nonzero elements in the cohomology of $A$, corresponding to permanent cycles in the May spectral sequence for $A$ as a filtered algebra (see [May 1966, Theorem 3] or [Weibel 1994, Theorem 5.4.1]). On page $E_{1}$ of this spectral sequence, their counterparts generate a polynomial ring over which $E_{1}$ is finitely generated (by the elements $1, \eta_{a}, \eta_{b}, \eta_{a} \eta_{b}$, where $\eta_{a}, \eta_{b}$ have cohomological degree 1 , functions dual to $a$ and $b$ in $\left.\operatorname{Hom}_{k}(\operatorname{gr} A, k) \cong \operatorname{Hom}_{\operatorname{gr} A}(\operatorname{gr} A \otimes \operatorname{gr} A, k)\right)$. The cohomology $\mathrm{H}^{*}(A, k)$ is finitely generated over its subalgebra generated by $\xi_{a}, \xi_{b}$, as a consequence of the proof of [Shroff 2013, Theorem 4.3]. We will see below that the subalgebra generated by $\xi_{a}, \xi_{b}$ is in fact a polynomial ring in $\xi_{a}, \xi_{b}$, which is Noetherian, so applying the left module version of [Goodearl and Warfield 2004, Corollary 1.5], $\mathrm{H}^{*}(A, k)$ is itself (left) Noetherian.

To verify the hypothesis of Theorem 3.1, we use the above information to define 2-cocycles representing elements in $\mathrm{H}^{*}(A \# k G, k)$ : Note that $a^{p}, b^{p}$ are $G$-invariant by [Cibils et al. 2009, (3.10)]. Thus, by the construction of $\xi_{a}, \xi_{b}$, these functions are also $G$-invariant, and so they in fact extend to 2-coboundaries on $B \# k G$, factoring through $A \# k G \cong B \# k G /\left(a^{p}, b^{p}\right)$. This also shows that $\xi_{a}, \xi_{b}$ commute with each other in $\mathrm{H}^{*}(A, k)$, since $\mathrm{H}^{*}(A \# k G, k)$ is graded commutative and $\xi_{a}, \xi_{b}$ each have even degree, so they are commuting elements in $\operatorname{Im}\left(\right.$ res $\left._{A \# k G, A}\right)$.

We next claim that $\xi_{a}, \xi_{b}$ generate a polynomial subalgebra $k\left[\xi_{a}, \xi_{b}\right]$ of $\mathrm{H}^{*}(A, k)$ over which $\mathrm{H}^{*}(A, k)$ is free with free basis $\left\{1, \eta_{a}, \eta_{b}, \eta_{a} \eta_{b}\right\} .{ }^{1}$ This will follow once we see that the set

$$
\left\{\xi_{a}^{i} \xi_{b}^{j} \eta_{a}^{l} \eta_{b}^{m} \mid i, j \geq 0, l, m=0,1\right\}
$$

represents a basis of $\mathrm{H}^{*}(A, k)$, since $\xi_{a}, \xi_{b}$ commute with each other. Note that the cohomology of $S=\operatorname{gr} A$ is well known, and has a basis precisely of this form. Recall that Anick's resolution for $A$ is minimal, and a comparison shows that in each degree, the dimensions of $\mathrm{H}^{*}(A, k)$ and of $\mathrm{H}^{*}(S, k)$ are the same. This forces the May spectral sequence [1966] for $A$ to collapse at $E_{1}=\mathrm{H}^{*}(S, k)$, and so $\operatorname{grH}^{*}(A, k) \cong \mathrm{H}^{*}(S, k)$, and $\mathrm{H}^{*}(A, k)$ has basis as claimed. This implies that $\xi_{a}, \xi_{b}$ generate a polynomial subring (we already know they commute). Therefore $\mathrm{H}^{*}(A, k)$ is free as a $k\left[\xi_{a}, \xi_{b}\right]$-module, as claimed. Further, the $k$-linear span of $\left\{1, \eta_{a}, \eta_{b}, \eta_{a} \eta_{b}\right\}$ is indeed a $k G$-submodule of $\mathrm{H}^{*}(A, k)$ : we compute

$$
{ }^{g} \eta_{a}=\eta_{a}+\eta_{b},{ }^{g} \eta_{b}=\eta_{b}, \quad{ }^{g}\left(\eta_{a} \eta_{b}\right)=\eta_{a} \eta_{b} .
$$

${ }^{1}$ Since $B$ is a Koszul algebra, $\mathrm{H}^{*}(B, k) \cong B^{!}$, the Koszul dual of $B$, which is generated by $\eta_{a}, \eta_{b}$ (by abuse of notation) with relations dual to those of $B$, that is, $\eta_{a}^{2}=\frac{1}{2} \eta_{a} \eta_{b}, \eta_{b}^{2}=0, \eta_{b} \eta_{a}=-\eta_{a} \eta_{b}$. These relations also hold in $\mathrm{H}^{*}(A, k)$, however we do not need this fact. 
We have shown that the hypotheses of Theorem 3.1 are satisfied. Therefore, $\mathrm{H}^{*}(A \# k G, k)$ is Noetherian.

Question 5.1. Are there more examples of Nichols algebras in positive characteristic to which Theorem 3.1 applies?

Nichols algebras and their bosonizations, which are Hopf algebras, have only just begun to be explored in positive characteristic. There is a vast (and recent) literature on Nichols algebras in characteristic zero. See, for example, [Andruskiewitsch et al. 2011a; 2011b; Andruskiewitsch and Schneider 2010; Heckenberger 2006].

\section{Acknowledgments}

We thank D. Benson and P. Symonds for very insightful conversations and suggestions. We thank $\varnothing$. Solberg for computing the cohomology of some of the Nichols algebras in Section 4; these computations led us to our general result on this series of Nichols algebras and corresponding Hopf algebras.

\section{References}

[Andruskiewitsch and Schneider 2010] N. Andruskiewitsch and H.-J. Schneider, "On the classification of finite-dimensional pointed Hopf algebras", Ann. of Math. (2) 171:1 (2010), 375-417. MR 2011j:16058 Zbl 1208.16028

[Andruskiewitsch et al. 2011a] N. Andruskiewitsch, F. Fantino, M. Graña, and L. Vendramin, "The logbook of pointed Hopf algebras over the sporadic simple groups”, J. Algebra 325 (2011), 282-304. MR 2012d:16092 Zbl 1217.16025

[Andruskiewitsch et al. 2011b] N. Andruskiewitsch, F. Fantino, M. Graña, and L. Vendramin, "Finitedimensional pointed Hopf algebras with alternating groups are trivial", Ann. Mat. Pura Appl. (4) 190:2 (2011), 225-245. MR 2012c:16095 Zbl 1234.16019

[Anick 1986] D. J. Anick, “On the homology of associative algebras”, Trans. Amer. Math. Soc. 296:2 (1986), 641-659. MR 87i:16046 Zbl 0598.16028

[Barnes 1985] D. W. Barnes, Spectral sequence constructors in algebra and topology, Mem. Amer. Math. Soc. 317, 1985. MR 86e:55032 Zbl 0557.18006

[Bueso et al. 2003] J. Bueso, J. Gómez-Torrecillas, and A. Verschoren, Algorithmic methods in non-commutative algebra: Applications to quantum groups, Mathematical Modelling: Theory and Applications 17, Kluwer, Dordrecht, 2003. MR 2005c:16069 Zbl 1063.16054

[Cibils et al. 2009] C. Cibils, A. Lauve, and S. Witherspoon, "Hopf quivers and Nichols algebras in positive characteristic", Proc. Amer. Math. Soc. 137:12 (2009), 4029-4041. MR 2010k:16047 Zbl 1191.16032

[Cojocaru and Ufnarovski 1997] S. Cojocaru and V. Ufnarovski, "BERGMAN under MS-DOS and Anick's resolution", Discrete Math. Theor. Comput. Sci. 1:1 (1997), 139-147. MR 99e:16017 Zbl 0935.16032

[Etingof and Ostrik 2004] P. Etingof and V. Ostrik, "Finite tensor categories", Mosc. Math. J. 4:3 (2004), 627-654. MR 2005j:18006 Zbl 1077.18005

[Evens 1961] L. Evens, "The cohomology ring of a finite group", Trans. Amer. Math. Soc. 101 (1961), 224-239. MR 25 \#1191 Zbl 0104.25101 
[Friedlander and Suslin 1997] E. M. Friedlander and A. Suslin, "Cohomology of finite group schemes over a field”, Invent. Math. 127:2 (1997), 209-270. MR 98h:14055a Zbl 0945.14028

[Ginzburg and Kumar 1993] V. Ginzburg and S. Kumar, "Cohomology of quantum groups at roots of unity”, Duke Math. J. 69:1 (1993), 179-198. MR 94c:17026 Zbl 0774.17013

[Goodearl and Warfield 2004] K. R. Goodearl and R. B. Warfield, Jr., An introduction to noncommutative Noetherian rings, 2nd ed., London Mathematical Society Student Texts 61, Cambridge University Press, 2004. MR 2005b:16001 Zbl 1101.16001

[Gordon 2000] I. G. Gordon, "Cohomology of quantized function algebras at roots of unity", Proc. London Math. Soc. (3) 80:2 (2000), 337-359. MR 2000m:20078 Zbl 1032.17025

[Heckenberger 2006] I. Heckenberger, "The Weyl groupoid of a Nichols algebra of diagonal type", Invent. Math. 164:1 (2006), 175-188. MR 2007e:16047 Zbl 1174.17011

[May 1966] J. P. May, "The cohomology of restricted Lie algebras and of Hopf algebras", J. Algebra 3 (1966), 123-146. MR 33 \#1347 Zbl 0163.03102

[Montgomery 1993] S. Montgomery, Hopf algebras and their actions on rings, CBMS Regional Conference Series in Mathematics 82, Amer. Math. Soc., Providence, RI, 1993. MR 94i:16019 Zbl 0793.16029

[Priddy 1970] S. B. Priddy, “Koszul resolutions”, Trans. Amer. Math. Soc. 152 (1970), 39-60. MR 42 \#346 Zbl 0261.18016

[Shroff 2013] P. Shroff, "Finite generation of the cohomology of quotients of PBW algebras", $J$. Algebra 390 (2013), 44-55. MR 3072110 Zbl 06266620

[Snashall and Solberg 2004] N. Snashall and Ø. Solberg, "Support varieties and Hochschild cohomology rings", Proc. London Math. Soc. (3) 88:3 (2004), 705-732. MR 2005a:16014 Zbl 1067.16010

[Suarez-Alvarez 2004] M. Suarez-Alvarez, "The Hilton-Heckmann argument for the anti-commutativity of cup products", Proc. Amer. Math. Soc. 132:8 (2004), 2241-2246. MR 2005a:18016 Zbl 1045.18002

[Weibel 1994] C. A. Weibel, An introduction to homological algebra, Cambridge Studies in Advanced Mathematics 38, Cambridge University Press, 1994. MR 95f:18001 Zbl 0797.18001

[Xu 2008] F. Xu, "Hochschild and ordinary cohomology rings of small categories", Adv. Math. 219:6 (2008), 1872-1893. MR 2009h:18025 Zbl 1156.18007

Communicated by Susan Montgomery

Received 2013-10-04

Accepted 2014-08-17

v.nguyen@neu.edu

Department of Mathematics, Northeastern University, 567 Lake Hall, Boston, MA 02115, United States

sjw@math.tamu.edu

Department of Mathematics, Texas A\&M University, Mailstop 3368, College Station, TX 77843, United States 


\section{Algebra \& Number Theory}

msp.org/ant

\section{EDITORS}

MANAGING EDITOR

Bjorn Poonen

Massachusetts Institute of Technology

Cambridge, USA

\author{
EDITORIAL BOARD CHAIR \\ David Eisenbud \\ University of California \\ Berkeley, USA
}

BOARD OF EDITORS

Georgia Benkart

Dave Benson

Richard E. Borcherds

John H. Coates

J-L. Colliot-Thélène

Brian D. Conrad

Hélène Esnault

Hubert Flenner

Edward Frenkel

Andrew Granville

Joseph Gubeladze

Roger Heath-Brown

Craig Huneke

Yujiro Kawamata

János Kollár

Yuri Manin

Barry Mazur

Philippe Michel

Susan Montgomery
University of Wisconsin, Madison, USA

University of Aberdeen, Scotland

University of California, Berkeley, USA

University of Cambridge, UK

CNRS, Université Paris-Sud, France

University of Michigan, USA

Freie Universität Berlin, Germany

Ruhr-Universität, Germany

University of California, Berkeley, USA

Université de Montréal, Canada

San Francisco State University, USA

Oxford University, UK

University of Virginia, USA

University of Tokyo, Japan

Princeton University, USA

Northwestern University, USA

Harvard University, USA

École Polytechnique Fédérale de Lausanne

University of Southern California, USA
Shigefumi Mori

Raman Parimala

Jonathan Pila

Anand Pillay

Victor Reiner

Peter Sarnak

Joseph H. Silverman

Michael Singer

Vasudevan Srinivas

J. Toby Stafford

Bernd Sturmfels

Richard Taylor

Ravi Vakil

Michel van den Bergh

Marie-France Vignéras

Kei-Ichi Watanabe

Efim Zelmanov

Shou-Wu Zhang
RIMS, Kyoto University, Japan

Emory University, USA

University of Oxford, UK

University of Notre Dame, USA

University of Minnesota, USA

Princeton University, USA

Brown University, USA

North Carolina State University, USA

Tata Inst. of Fund. Research, India

University of Michigan, USA

University of California, Berkeley, USA

Harvard University, USA

Stanford University, USA

Hasselt University, Belgium

Université Paris VII, France

Nihon University, Japan

University of California, San Diego, USA

Princeton University, USA

\section{PRODUCTION}

production@msp.org

Silvio Levy, Scientific Editor

See inside back cover or msp.org/ant for submission instructions.

The subscription price for 2014 is US $\$ 225 /$ year for the electronic version, and $\$ 400 /$ year ( $\$ 55$, if shipping outside the US) for print and electronic. Subscriptions, requests for back issues and changes of subscribers address should be sent to MSP.

Algebra \& Number Theory (ISSN 1944-7833 electronic, 1937-0652 printed) at Mathematical Sciences Publishers, 798 Evans Hall \#3840, c/o University of California, Berkeley, CA 94720-3840 is published continuously online. Periodical rate postage paid at Berkeley, CA 94704, and additional mailing offices.

ANT peer review and production are managed by EditFLOW ${ }^{\circledR}$ from MSP.

\section{PUBLISHED BY}

- mathematical sciences publishers

nonprofit scientific publishing

http://msp.org/

(C) 2014 Mathematical Sciences Publishers 


\section{Algebra \& Number Theory}

Volume $8 \quad$ No. $7 \quad 2014$

Double Dirichlet series and quantum unique ergodicity of weight one-half Eisenstein series

Yiannis N. Petridis, Nicole RaUlF and Morten S. Risager

Monodromy and local-global compatibility for $l=p$

ANA CARAIANI

Finite generation of the cohomology of some skew group algebras

VAN C. NGUYEN and SARAH WITHERSPOON

On the supersingular locus of the $G U(2,2)$ Shimura variety

BENJAMIN HOWARD and GEORgIOS PAPPAS

Poincaré-Birkhoff-Witt deformations of smash product algebras from Hopf actions on

1701

Koszul algebras

Chelsea Walton and Sarah Witherspoon

Highly biased prime number races

1733

DANIEL FIORILLI

Bounded gaps between primes with a given primitive root

1769

PAUL POLlACK 